\title{
Funicular state in drying of a porous slab
}

\author{
M. KAVIANY \\ Department of Mechanical Engineering and Applied Mechanics, University of Michigan, \\ Ann Arbor, MI 48109, U.S.A. \\ and \\ M. MITTAL \\ Department of Mechanical Engineering, University of Wisconsin-Milwaukee, \\ Milwaukee, WI 53201, U.S.A.
}

(Received $8 \mathrm{July} 1986$ and in final form 17 Nowember 1986)

\begin{abstract}
Drying of a non-hygroscopic porous slab initially saturated with liquid, up to the time of the first appearance of dried patches (critical time), is studied experimentally using glass beads and convective heating, and analytically using the volume averaged conservation equations for capillary driven liquid flow and empirical constitutive relations. Good agreement has been found between the predicted and measured results and the results show that:
\end{abstract}

(a) The surface mass transfer rate depends on the surface saturation. This surface saturation coefficient, which is also a function of both surface geometry and the free stream velocity, is determined experimentally.

(b) The critical time is a ratio of the internal liquid transport conductance (Péclet number) to the external vapor transport conductance (Biot number).

\section{INTRODUCTION}

IN REMOVING liquids from porous media two regimes are identified [1-3]. During the early stages where the entrapped liquid is mobile (funicular state), the drying rate is ncarly constant; this is followed by a falling rate period where the liquid is immobile (pendular state). Conventionally the transition from the funicular state to the pendular state is marked by a critical average liquid saturation. However, it has been observed that the drying rate begins to drop when dried patches start to appear on the surface. The time of this appearance (here called the critical time) depends on the intensity of the drying and on the medium permeability, rather than the average liquid saturation. After the critical time has elapsed, the surface temperature and saturation fluctuate due to the periodic rewetting of the surface. However, on the average, the surface temperature increases with time and the drying rate decreases. This, in turn, is followed by the pendular state in which the drying proceeds as a combination of internal evaporation-condensation and a moving interface.

For a given porous medium and drying condition, it is desirable to predict the time at which the surface begins to dry, because after this elapsed time the surface temperature increases rapidly to levels which hinder heat transfer to the porous medium and may also cause irreversible damage to the solid matrix.

Drying in the funicular state has been previously studied in refs. [4-6], where in ref. [6] the anisotropic structure of a certain wood was modelled and the drying mechanism was categorized as internal, transition and external, for high, intermediate and low permeabilities, respectively. Both funicular and pendular states have been investigated in ref. [7] where constant conductances were assumed for the heat and mass transport. As will be shown, these conductances are strong functions of local liquid saturation. The pendular state has been studied for moderate heating rates in refs. $[8,9]$ and for intense heating rates in refs. $[10,11]$. However, the concept of critical time has not been studied extensively before.

The process of liquid, vapor and thermal energy transport in porous media can be described by the conservation principles, provided the proper constitutive equations, which are presently mostly empirical, are available. These include physical descriptions of both internal (i.e. solid-liquid-gas interstitial phenomena) transports as well as the surface transports.

In this study, drying of non-consolidated, nonhygroscopic isotropic porous media in the funicular state and up to the time of the first appearance of dried patches on the surface (critical time) is considered experimentally using glass beads and also analytically by applying the conservation principles and empiricism. Figure 1 gives a schematic of the problem considered.

\section{ANALYSIS}

Descriptions of the conservation of mass, momentum and thermal energy for porous media saturated 


\section{NOMENCLATURE}

$A, B, C$ constants in equation (25)

$A_{\mathrm{p}}$ plate area $\left[\mathrm{m}^{2}\right]$

$a, b$ constants in the vapor pressure equation

$B i \quad$ Biot number, $h L / k_{\sigma-\beta}$

c specific heat capacity $\left[\mathrm{J} \mathrm{kg}^{-1} \mathrm{~K}^{-1}\right]$

$d$ bead diameter [m]

$D$ mass diffusion coefficient (water vapor in air) $\left[\mathrm{m}^{2} \mathrm{~s}^{-1}\right]$

$f \quad$ saturation function in the capillary pressure equation

$h_{\mathrm{h}} \quad$ average heat transfer coefficient $\left[\mathrm{W} \mathrm{m}{ }^{-2} \mathrm{~K}^{-1}\right]$

$i_{\mathrm{Ig}} \quad$ heat of evaporation $\left[\mathrm{J} \mathrm{kg}^{-1}\right]$

$I$ current supplied to the individual strip heaters $[\mathrm{A}]$

$G$ internal heat absorption $\left[\mathrm{W} \mathrm{m}^{-3}\right]$

$k$ thermal conductivity $\left[\mathrm{W} \mathrm{m}^{-1} \mathrm{~K}^{-1}\right]$

$K \quad$ permeability $\left[\mathrm{m}^{2}\right]$

$K_{r \beta} \quad$ liquid relative permeability

$K_{\mathrm{r} \gamma} \quad$ vapor relative permeability

$L \quad$ layer length [m]

Le Lewis number, $D \rho_{1} c_{1} / k_{1}$

$m \quad$ mass flux $\left[\mathrm{kg} \mathrm{m}^{-2} \mathrm{~s}^{-1}\right]$

$M \quad$ molecular weight $\left[\mathrm{kg} \mathrm{kg} \mathrm{mol}^{-1}\right]$

$\dot{n}$ volumetric vapor generation $\left[\mathrm{kg} \mathrm{m}^{-3} \mathrm{~s}^{-1}\right]$

$p \quad$ pressure $\left[\mathrm{N} \mathrm{m}^{-2}\right]$

$P e ́$ Péclet number, $\varepsilon \sigma(K / \varepsilon)^{1 / 2} / \alpha_{\beta} \mu_{\beta}$

$q$ e external heat flux [ $\mathrm{W} \mathrm{m}^{-2}$ ]

$Q_{\mathrm{p}}$ total heat flow rate through the plate [W]

$R_{\gamma}$ gas constant for vapor $\left[\mathrm{J} \mathrm{kg}^{-1} \mathrm{~K}^{-1}\right]$

$s \quad$ saturation, $\varepsilon_{\beta} / \varepsilon$

$\bar{s} \quad \int_{0}^{1} s \mathrm{~d} z$

$S \quad\left(s-s_{\mathrm{im}}\right) /\left(1-s_{\mathrm{im}}\right)$

$t$ time [s] $t_{c} \quad$ critical time [s]

$T$ temperature [K]

$u \quad$ velocity $\left[\mathrm{m} \mathrm{s}^{-1}\right]$

$V \quad$ voltage supplied to individual strip heaters

[V]

$z \quad$ coordinate axis $[\mathrm{m}]$.

Greek symbols

$\alpha \quad$ thermal diffusivity, $k_{\mathrm{e}} /(\rho c)_{\beta}\left[\mathrm{m}^{2} \mathrm{~s}^{-1}\right]$

$\varepsilon \quad$ porosity

$\mu \quad$ viscosity $\left[\mathrm{kg} \mathrm{m}^{-1} \mathrm{~s}^{-1}\right]$

$\rho$ density $\left[\mathrm{kg} \mathrm{m}^{-3}\right]$

$\sigma \quad$ surface tension $\left[\mathrm{N} \mathrm{m}^{-1}\right]$

$\Psi$ surface saturation coefficient.

Superscripts

* quantity is dimensional average.

Subscripts

0 reference

1 evaluated at the surface

a ambient

c capillary

D Darcy's

e effective

exp experimental

h heat

im immobile liquid saturation

m mass

p plate

s surface

sat saturation

$\beta \quad$ liquid phase

$\gamma \quad$ vapor phase

$\sigma \quad$ solid phase. by a liquid, its vapor and noncondensables, are given in ref. [12]. This includes the concepts of: (i) capillary pressure, (ii) relative permeabilities, (iii) vapor press-

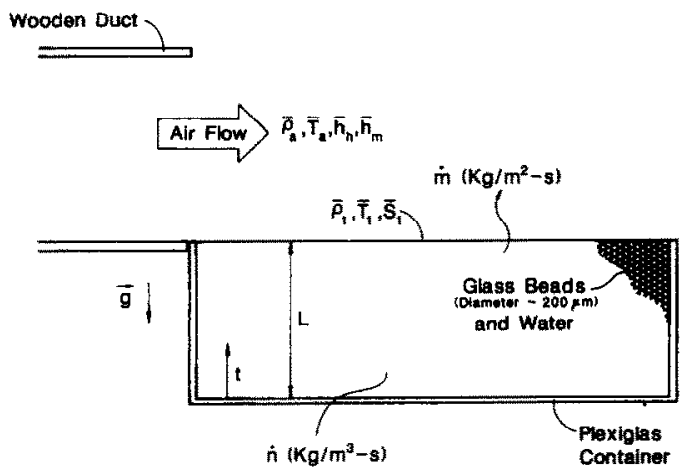

FIG. 1. A schematic of the problem considered. ure reduction, and (iv) diffusion of the vapor in the noncondensable gas (or vice versa). For moderate drying intensities and during the early stages of drying, the vapor transport is not significant and the noncondensables only influence the surface mass transfer rate. In the following, for the sake of completeness, the vapor transport in porous media is included in the analysis and is described through the appropriate momentum and mass conservation equations. However, no allowance is made for the internal resistance due to the diffusion of the vapor through the noncondensables.

\subsection{Governing equations}

2.1.1. Conservation equations. The following onedimensional conservation equations are those given in ref. [12] and are nondimensionalized using the liquid 
Table 1. Dimensionless variables

\begin{tabular}{cccccccccc}
\hline$z$ & $t$ & $u_{\beta}$ & $u_{i}$ & $T$ & $\dot{n}$ & $s$ & $g$ & $\rho_{i}$ & $p_{7}$ \\
\hline$\frac{z^{*}}{L}$ & $\frac{t^{*} \alpha_{\beta}}{L^{2}}$ & $\frac{u_{\beta}^{*} L}{\alpha_{\beta}}$ & $\frac{u_{i}^{*} L}{\alpha_{\beta}}$ & $\frac{T^{*}}{T_{0}}$ & $\frac{\dot{n}^{*} L^{2}}{\rho_{\beta}^{*} \alpha_{\beta}}$ & $\frac{\varepsilon_{\beta}}{\varepsilon}$ & $\frac{g^{*} L^{2}}{k_{\sigma-\beta}^{*} T_{0}}$ & $\frac{\rho_{i}^{*}}{\rho_{\beta}^{*}}$ & $\frac{p_{\gamma}^{*}(K / \varepsilon)^{1 / 2}}{\sigma}$ \\
\hline
\end{tabular}

Table 2. Dimensionless parameters

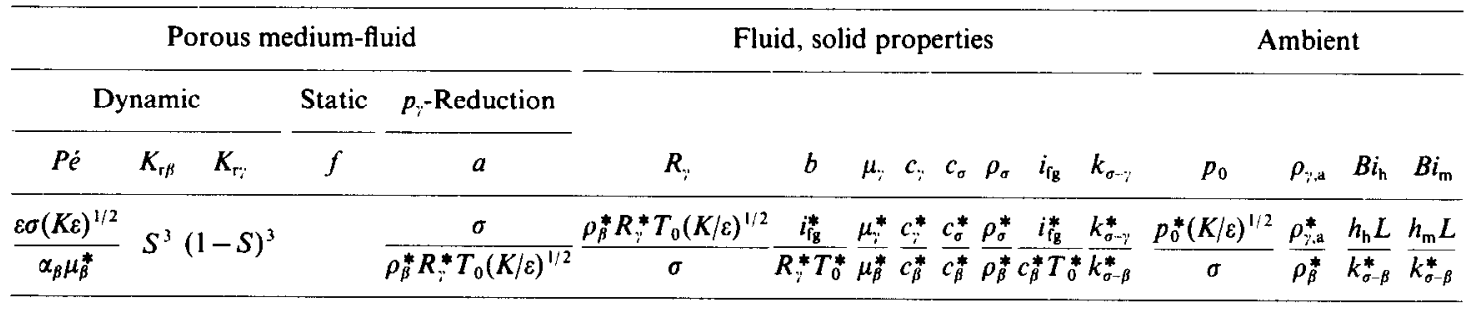

phase properties. The liquid is driven by the capillary pressure gradient while the vapor is driven by the gradient of the partial pressure of the evaporating species. The dimensionless variables and parameters are given in Tables 1 and 2. The symbols are defined in the Nomenclature. Dimensional quantities are shown with asterisks. The coordinate system is shown in Fig. 1. Perfect gas behavior is assumed for the vapor and the noncondensables. The ratio of the gravity force per unit volume to the gradient of the capillary pressure can be approximated by $g \rho_{\beta}^{*}(K / \varepsilon)^{1 / 2} L / \sigma$. In this study the effect of the gravity field is neglected, but as expected for higher permeabilities and for very small saturation gradients this is not justifiable.

Dimensionless liquid phase momentum $\dagger$ conservation equation

$$
u_{\beta}=-P \dot{e} s K_{\mathrm{r} \beta} \frac{\partial p_{\mathrm{c}}}{\partial z} .
$$

Dimensionless vapor phase momentum conservation equation

$$
u_{\gamma}=-\frac{P \dot{e}(1-s) K_{\mathrm{r} \gamma}}{\mu_{\gamma}} \frac{\partial p_{\gamma}}{\partial z} .
$$

Dimensionless energy conservation equation, including an internal heat absorption (microwave, etc.) proportional to the local liquid saturation (i.e. Gs)

$$
\begin{aligned}
\frac{\partial}{\partial z}\left(k_{\mathrm{e}} \frac{\partial T}{\partial z}\right)-i_{\mathrm{fg}} \dot{n}+G s= & {\left[(1-\varepsilon)(\rho c)_{\sigma}\right.} \\
& \left.+\varepsilon s+\varepsilon(1-s)(\rho c)_{\gamma}\right] \frac{\partial T}{\partial t} .
\end{aligned}
$$

For simplicity, the convection heat transfer through the liquid and vapor phases is neglected, where for the drying intensity considered and in the initial stages of drying only the former can become significant. The

† The velocities are the superficial velocities [12], i.e. these can be used directly for the determination of the flow rates. relative significance of the liquid phase convection heat transfer can be estimated by the magnitude of $P e ́ s^{4}$, which indicates that for relatively high permeabilities and saturations, convection can become significant.

Dimensionless liquid phase mass conservation equation

$$
-P \dot{e} \frac{\partial}{\partial z}\left(s K_{\mathrm{r} \beta} \frac{\mathrm{d} f}{\mathrm{~d} s} \frac{\partial s}{\partial z}\right)-\dot{n}=\varepsilon \frac{\partial s}{\partial t} .
$$

Dimensionless vapor phase mass conservation equation (based on perfect gas behavior)

$$
\begin{aligned}
\frac{R_{\gamma} P e ́}{\mu_{\gamma}} \frac{\partial}{\partial z}[ & (1-s) \rho_{y} K_{\mathrm{r \gamma}} T \frac{\partial \rho_{\gamma}}{\partial z} \\
& \left.+(1-s) K_{\mathrm{r} \gamma} \rho_{\gamma}^{2} \frac{\partial T}{\partial z}\right]+\dot{n}=\varepsilon \frac{\partial}{\partial t}(1-s) \rho_{\gamma}
\end{aligned}
$$

Now adding equations (4) and (5), the overall continuity equation for the evaporating species, i.e. saturation equation, emerges

$$
\begin{aligned}
-P \dot{e} \frac{\partial}{\partial z}\left(s K_{\mathrm{r} \beta} \frac{\mathrm{d} f}{\mathrm{~d} s} \frac{\partial s}{\partial z}\right)+\frac{R_{\gamma} P \dot{e}}{\mu_{\gamma}} \frac{\partial}{\partial z} \\
\times\left[(1-s) \rho_{\gamma} K_{\mathrm{r} \gamma} T \frac{\partial \rho_{\gamma}}{\partial z}+(1-s) K_{\mathrm{r} \gamma} \rho_{\gamma}^{2} \frac{\partial T}{\partial z}\right] \\
=\varepsilon\left(1-\rho_{\gamma}\right) \frac{\partial s}{\partial t}+\varepsilon(1-s) \frac{\partial \rho_{\gamma}}{\partial t} .
\end{aligned}
$$

Also, substituting for $\dot{n}$ using equation (5), the energy conservation equation becomes

$$
\begin{gathered}
\frac{\partial}{\partial z}\left(k_{\mathrm{e}} \frac{\partial T}{\partial z}\right)+i_{\mathrm{fg}}\left\{-\varepsilon \frac{\partial}{\partial t}(1-s) \rho_{\gamma}+\frac{R_{\gamma} P \dot{e}}{\mu_{\gamma}} \frac{\partial}{\partial z}\right. \\
\left.\times\left[(1-s) \rho_{\gamma} K_{\mathrm{r \gamma}} T \frac{\partial \rho_{\gamma}}{\partial z}+(1-s) K_{\mathrm{r}_{\gamma}} \rho_{\gamma}^{2} \frac{\partial T}{\partial z}\right]\right\}+G s \\
=\left[(1-\varepsilon)(\rho c)_{\sigma}+\varepsilon s+\varepsilon(1-s)(\rho c)_{\gamma}\right] \frac{\partial T}{\partial t} .
\end{gathered}
$$


2.1.2. Boundary and initial conditions. The physical problem considered is a porous slab that is initially completely saturated with liquid while at a uniform temperature. Then one side of the slab is convectively heated while the other side $(z=0)$ is in contact with an impermeable and ideally insulating surface (Fig. 1). Stated mathematically, the initial conditions are $T(t=0)=T_{i}$ and $s(t=0)=1$. In addition to $T_{z}=s_{z}=0$ at $z=0$, the two boundary conditions at the convection interface are :

surface mass balance

$$
\begin{gathered}
P \dot{e}\left(s K_{\mathrm{r} \beta} \frac{\mathrm{d} f}{\mathrm{~d} s} \frac{\partial s}{\partial z}\right)-P \dot{e} K_{\mathrm{r} \gamma} \frac{R_{\gamma}}{\mu_{\gamma}} T \rho_{\gamma}(1-s) \frac{\partial \rho_{\gamma}}{\partial z} \\
-P \dot{e} K_{\mathrm{r} \gamma} \frac{R_{\gamma}}{\mu_{\gamma}} \rho_{\gamma}^{2}(1-s) \frac{\partial T}{\partial z}=\dot{m}_{\beta}+\dot{m}_{\gamma} \\
=\frac{B i_{\dot{m}}}{\rho_{\mathrm{sat}} c_{\gamma}}\left(\rho_{\gamma}-\rho_{\gamma, \mathrm{a}}\right)=\dot{m} ;
\end{gathered}
$$

surface heat balance

$$
\begin{array}{rl}
B i_{\mathrm{h}}\left(T_{\mathrm{a}}-T\right)=q_{\mathrm{e}}=i_{\mathrm{f}} & P \dot{e}\left(s K_{\mathrm{r} \beta} \frac{\mathrm{d} f}{\mathrm{~d} s} \frac{\partial s}{\partial z}\right) \\
& +\left[k_{\sigma-\gamma}+s^{1 / 2}\left(1-k_{\sigma-\gamma}\right)\right] \frac{\partial T}{\partial z} .
\end{array}
$$

2.1.3. Constitutive relations. The following empirical relations, some of which have already been used in the above conservation equations, describe the various properties of porous media.

Saturation vapor pressure equation, including reduction due to capillarity [12]

$$
p_{\gamma}=R_{\gamma} \rho_{\gamma} T=p_{0} \exp \left[-\frac{a f}{T}-b\left(\frac{1}{T}-1\right)\right]
$$

\section{Capillary pressure [13]}

$$
\begin{aligned}
p_{\mathrm{c}}^{*}= & \frac{\sigma}{(K / \varepsilon)^{1 / 2}}\left[1.417(1-S)-2.120(1-S)^{2}\right. \\
& \left.+1.263(1-S)^{3}\right]=\frac{\sigma}{(K / \varepsilon)^{1 / 2}} f(S)=p_{\nu}^{*}-p_{\beta}^{*}
\end{aligned}
$$

where

$$
S=\frac{s-s_{\mathrm{im}}}{1-s_{\mathrm{im}}}
$$

also

$$
\frac{\mathrm{d} f}{\mathrm{~d} s}=\frac{\mathrm{d} f}{\mathrm{~d} S} \frac{\mathrm{d} S}{\mathrm{~d} s}=\frac{1}{1-s_{\mathrm{im}}} \frac{\mathrm{d} f}{\mathrm{~d} S}
$$

As was mentioned before, asterisks indicate that the quantities are dimensional.

Relative permeability (based on the results obtained

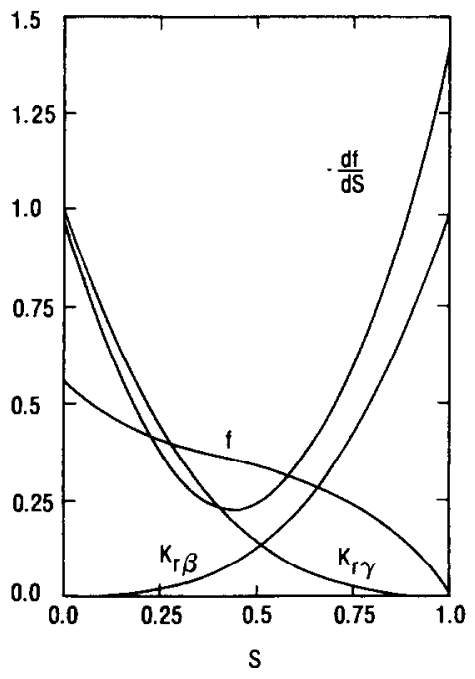

Fig. 2. Variations in the functions related to relative permeability and the capillary pressure with respect to normalized saturation.

in this study and as suggested in ref. [13])

$$
K_{\mathrm{r} \beta}=S^{3}, \quad K_{\mathrm{ry}}=(1-S)^{3} .
$$

Figure 2 shows the variations of $f, \mathrm{~d} f / \mathrm{d} S, K_{\mathrm{r} \beta}$ and $K_{r y}$. Effective thermal conductivity [13] is

$$
k_{\mathrm{e}}^{*}=k_{\sigma-\gamma}^{*}+s^{1 / 2}\left(k_{\sigma-\beta}^{*}-k_{\sigma-\gamma}^{*}\right) .
$$

2.1.4. Overall balance. The following balance equations are obtained by integrating the mass and energy conservation equations over the length of the slab and also over a time interval.

Overall mass balance

$$
\int_{0}^{t} \int_{0}^{1}\left[\varepsilon \frac{\partial s}{\partial t}+\varepsilon \frac{\partial}{\partial t}(1-s) \rho_{\gamma}\right] \mathrm{d} z \mathrm{~d} t=\int_{0}^{t} \dot{m} \mathrm{~d} t .
$$

Overall energy balance

$$
\begin{gathered}
\int_{0}^{t} \int_{0}^{1}\left\{\left[(1-\varepsilon)(\rho c)_{\sigma}+\varepsilon s+\varepsilon(1-s)(\rho c)_{\gamma}\right] \frac{\partial T}{\partial t}\right. \\
-G s\} \mathrm{d} z \mathrm{~d} t=\int_{0}^{t}\left[B i_{\mathrm{h}}\left(T_{\mathrm{a}}-T_{\mathrm{l}}\right)\right. \\
\left.-\dot{m}_{\gamma} i_{\mathrm{fg}}-\dot{m}_{\beta}\left(T_{1}-T_{i}+i_{\mathrm{fg}}\right)\right] \mathrm{d} t .
\end{gathered}
$$

2.1.5. Mass transfer coefficient. The mass transfer coefficient can be determined (by analogy) from the heat transfer coefficient [14, 15]. Following [14] from the analogy between forced convection heat and mass transfers (page 647 of ref. [14]), the relationship between the heat and mass transfer coefficients is $h_{\mathrm{m}}=h_{\mathrm{h}} \Psi\left(c_{\gamma} / c_{1}\right)\left(M_{\mathrm{H}_{2} \mathrm{O}} / \bar{M}\right) L e^{2 / 3}$, where $c_{1}$ is the mass averaged specific heat capacity, $\bar{M}$ is the mass averaged molecular weight of the air-water-vapor mixture, and $L e$ is the Lewis number. All are evaluated at the film temperature and concentration. The effec- 
tive area available for convective mass transfer between the entrapped liquid and the ambient can be expressed as a fraction of the plane interface. This fraction depends on the surface saturation. This effective surface area is given by $\Psi$, the surface saturation coefficient, and needs to be determined experimentally. The values obtained in this study will be given in the following section. In general, this coefficient depends also on both the surface geometry and air flow velocity, i.e. $\Psi=\Psi\left(s_{1}\right.$, geometry, $\left.u\right)$. The details of this functional relationship are not yet available. A similar coefficient has been used in ref. [6] for a certain wood (hygroscopic).

\subsection{Solution}

The partial differential equations are solved numerically using second-order finite-difference approximation forms for spatial derivatives and first-order forward forms for the temporal derivatives. The explicit technique was applied and the corresponding stability criterion was satisfied by choosing the proper combination of $\Delta z$ and $\Delta t$. The standard convergence technique of using progressively smaller grid sizes was satisfactorily implemented. A compromise between accuracy and computer economy was made by using $\Delta z=0.05$ and the corresponding stable $\Delta t$. For example, in the case of $h_{\mathrm{h}}=50 \mathrm{~W} \mathrm{~m} \mathrm{~m}^{-2} \mathrm{~K}^{-1}$ and for $K=10^{-11} \mathrm{~m}^{2}, \Delta t=10^{-6}$ was required, and for the case of $K=10^{-12} \mathrm{~m}^{2}$, the required $\Delta t$ was $4 \times 10^{-6}$. The computation time for high permeabilities becomes very excessive. Overall heat and mass balances were performed for each time step, requiring agreement of less than $1 \%$ for all the results reported.

The surface temperature begins to fluctuate when the surface begins to dry (critical time). This takes place when the liquid saturation near the surface is so low such that the liquid flow rate can no longer maintain a non-zero saturation at the surface. Note that the resistance to the liquid flow is proportional to $s^{4}$ and this resistance becomes significant as $s$ decreases substantially. Consequently, the surface temperature increases. The resulting large saturation gradient will then cause rewetting of the surface. This is followed by an increase in the evaporation rate at the surface and, consequently, the surface saturation decreases again and a periodic rewetting-evaporation takes place. In the computations, whenever the surface saturation dropped significantly, as a result of time step $\Delta t$, leading to surface dryout, the computation was repeated starting from the results at $t-\Delta t$, but with time steps much smaller than $\Delta t$. It was always observed that the surface dryout was not due to the time step size. In the experiments that will be discussed later, it was not possible to observe these rather fast fluctuations in the surface temperature. The only experimental evidence of the critical time is the rather rapid drop in the heating rate observed after the predicted critical time. The present analysis is limited to the elapsed times up to the critical time. A different formulation $[3,11]$ is needed in order to extend the analysis to the later periods. The computations were discontinued once surface liquid saturation became zero.

\section{EXPERIMENT}

In order to obtain the relationships required in the analysis, experiments were performed for determination of : (a) the relative permeability of air flowing through a partially liquid filled packed bed made of glass beads, and (b) the external heat transfer coefficient. Then, drying tests were conducted which resulted in the determination of the surface saturation coefficient and the drying rate.

\subsection{Measurement of relative permeability of air}

The relative permeability of a partially liquid saturated packed bed is measured using air as the flowing fluid and water as the stagnant liquid.

3.1.1. Partially saturating the packed bed. The sctup for this consisted of the following :

(i) a plexiglas tube $31.75 \mathrm{~cm}$ in length and $2.54 \mathrm{~cm}$ in inner diameter,

(ii) two cylindrical pieces of polyurethane foam each $1.91 \mathrm{~cm}$ in length and $2.54 \mathrm{~cm}$ in diameter, and

(iii) glass beads with an average diameter of 210 $\mu \mathrm{m}$.

A piece of polyurethane foam was placed at a distance of $4.00 \mathrm{~cm}$ from one end of the tube. A measured volume $\left(10.8 \mathrm{~cm}^{3}\right)$ of dry glass beads was poured at this end. A second piece of foam was then placed on top of the glass beads, so that the glass beads were enclosed between the two pieces of foam. This assembly was weighed $(w \mathrm{~g})$ to a precision of $0.001 \mathrm{~g}$.

The assembly was placed in a vertical position with the foams and the glass beads on the top. Then the upper piece of foam was removed and the bottom of the tube was connected to a vessel which contained boiling water. The duration of steam passage through the glass beads was controlled according to the required saturation. The steam condensed while flowing through the glass beads. When the steam passage was stopped, the top foam was repositioned and the assembly weighed again $(y \mathrm{~g})$.

The liquid saturation is defined as

$$
s=\frac{\varepsilon_{\beta}}{\varepsilon}
$$

where

$$
\varepsilon_{\beta}=\frac{y-w}{\rho_{\beta} v} .
$$

Here $\varepsilon$ is the porosity (measured to be about 0.40 ), $\rho_{\beta}$ is the liquid density (taken as $1 \mathrm{~g} \mathrm{~cm}^{-1}$ ) and $v$ is the volume of the packed bed (equal to $10.8 \mathrm{~cm}^{3}$ ).

3.1.2. Measurement of permeability. The permeability for air (as the gas phase) flow through the porous medium was measured as follows. The entire 
assembly was connected to another plexiglas tube, which was connected to a water manometer and an air flow meter. Pressure drop across the foams and glass beads was measured for a flow rate of $51 \mathrm{~min}^{-1}$. The foams have permeabilities of the order of $10^{-7}$ $\mathrm{m}^{2}$ which is much larger than the permeabilities obtained for the packed bed. Therefore, the pressure drop measured is taken to be due to the packed bed.

Based on the general form of equation (2), the permeability is conventionally determined using

$$
(1-s) \frac{\mathrm{d} p_{y}^{*}}{\mathrm{~d} z^{*}}=-\frac{\mu_{y}^{*}}{K_{\gamma}} u_{y}^{*}-\rho_{\gamma}^{*} \frac{F}{K_{y}^{1 / 2}} u_{\gamma}^{* 2}
$$

However, due to the experimental limitations, and since at high velocities the liquid was removed from the packed bed by interfacial shear stress, no satisfactory results were obtained for relatively high flow rates. For relatively small velocities, the second term in equation (18) is negligible and the above expression reduces to

$$
\frac{\mathrm{d} p_{\gamma}^{*}}{\mathrm{~d} z^{*}} \simeq-\frac{\mu_{\gamma}^{*} u_{\gamma}^{*}}{K_{y}(1-s)}
$$

For validation, random samples of glass beads were taken and diameters of 34 beads were measured under a microscope. A large number of beads had diameters in the range of $0.22-0.24 \mathrm{~mm}$. The histogram tapered sharply for diameters greater than $0.25 \mathrm{~mm}$ and gradually for diameters less than $0.21 \mathrm{~mm}$. The weighted mean diameter was calculated to be 0.21 $\mathrm{mm}$. An estimate of the permeability was made by using the following empirical relationship :

$$
K=\frac{\varepsilon^{3} d^{2}}{150(1-\varepsilon)^{2}}
$$

For $d=0.21 \mathrm{~mm}, K=5.23 \times 10^{-11} \mathrm{~m}^{2}$. Experiments were performed with dry beads. The experimental results showed a value of $K=4.43 \times 10^{-11} \mathrm{~m}^{2}$. The predicted and measured permeabilities were fairly close. Based on this validation, the measured relative permeabilities were accepted as reasonable.

3.1.3. Relative permeability. The effective gas permeability $\left(K_{\gamma}\right)$ of partially liquid saturated media is expressed as a fraction of the permeability $(K)$ and is known as the relative permeability, this is

$$
K_{r y}=\frac{K_{y}}{K^{\circ}}
$$

In Fig. 3, the measured $K_{r}$ has been plotted against a normalized saturation $S$ defined as

$$
S=\frac{s-s_{\mathrm{im}}}{1-s_{\mathrm{im}}}
$$

where $s$ is the absolute saturation and $s_{\text {im }}$ is the 'immobile' or 'residual' saturation. Since an experimental value for $s_{\mathrm{im}}$ was not available, a value of 0.1 was assumed. It should be noted that the results of ref. [16] show that in some cases a significant error in determining $s_{\mathrm{im}}$ can lead to meaningless predictions.

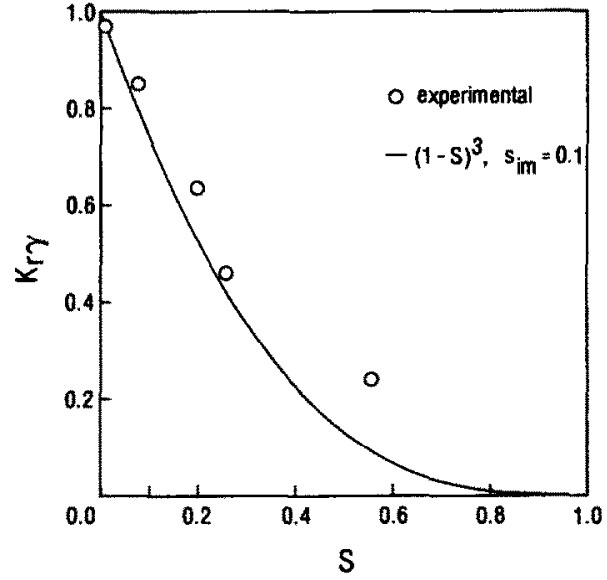

FIG. 3. The measured relative permeability for air flowing in a partially liquid saturated glass bead packed bed. The solid line is an approximation to the measured results.

The empirical results are also shown in the same graph. The empirical expression is

$$
K_{r y}=(1-S)^{3} \text {. }
$$

\subsection{Determination of heat transfer coefficient}

The heat transfer coefficient, $h_{\mathrm{h}}$, for forced convection over the glass bead surface, was determined by replacing the porous slab with a heated plate coated by a few layers of glass beads to simulate the rough surface. The setup consisted of the following:

(i) A wooden channel, $20 \mathrm{~cm}$ (width) $\times 10 \mathrm{~cm}$ (height) cross-section, a fan mounted at one end and two $600 \mathrm{~W}$ heaters placed inside the channel.

(ii) An aluminum plate, $20 \mathrm{~cm}$ (width) $\times 15 \mathrm{~cm}$ (length) $\times 0.3 \mathrm{~cm}$ (height), with ten strip heaters placed underneath it and ten thermocouples centered at equal spaces along the $15 \mathrm{~cm}$ side. The plate-heater assembly was placed in an indented area of a marinite block such that the aluminum surface was flush with the marinite.

(iii) Ten signal conditioning circuits, a HP data acquisition unit and a HP 9817 computer.

(iv) Glass beads (weighted mean diameter of 0.21 $\mathrm{mm}$ ).

To simulate the actual packed bed surface, a very thin layer of hair spray (or any volatile liquid which leaves small deposits upon evaporation) was sprayed over the aluminum plate. Then, the glass beads were sprinkled over the plate, such that they formed a thin uniform layer (about two beads thick). This was done to prevent the glass beads from detaching from the surface by the air currents. After the hair spray had evaporated, the plate was placed in front of the open end of the channel, and the fan was turned on. The strip heaters were connected to a 110 a.c. input, variable transformer through the signal conditioning circuits. The voltages across the strip heaters and the temperature of the aluminum plate were monitored. With the help of the signal conditioning circuits, the 
voltage across each strip heater was adjusted so that the temperature of the aluminum plate became uniform (this required readjustments every $10 \mathrm{~min}$ ). The system was allowed to reach a steady state, which took approximately $30-90 \mathrm{~min}$.

The total heat transfer, $Q_{\mathrm{p}}$, from the bead covered surface was calculated by the power input into the heaters, i.e.

$$
Q_{\mathrm{p}}=\sum_{j=1}^{10}\left(V_{j} I_{j}\right) .
$$

It was assumed that the temperature of the bead surface was equal to the temperature of the aluminum plate, $T_{\mathrm{p}}$. The heat losses through the marinite were also assumed to be negligible.

The average heat transfer coefficient, $h_{\mathrm{h}}$, was then calculated by

$$
h_{\mathrm{h}}=\frac{Q_{\mathrm{p}}}{\left(T_{\mathrm{p}}-T_{\mathrm{a}}\right) A_{\mathrm{p}}} .
$$

This procedure was repeated for three fan speeds, which resulted in $h_{\mathrm{h}}$ values of $30.9,40.0$, and $51.9 \mathrm{~W}$ $\mathrm{m}^{-2} \mathrm{~K}^{-1}$. Since the air velocity was not measured, no comparison could be made with the available results.

\subsection{Experiment on drying of packed bed}

The setup was the same as that described above except that the aluminum plate was replaced by a container full of completely saturated glass beads with the power to channcl heaters turned on.

A rectangular plexiglas container (inside dimensions : $15 \mathrm{~cm}$ (width) $\times 15 \mathrm{~cm}$ (length) $\times 5 \mathrm{~cm}$ (height) with one of the $15 \mathrm{~cm} \times 15 \mathrm{~cm}$ surfaces open, was filled with a known amount of water (equal in volume to the void volume). Glass beads were slowly poured into the container, with regular stirring to remove air bubbles, until the container was completely filled. This container also had three thermocouples placed in the center and at distances of $0,2.5$ and $5 \mathrm{~cm}$ from the bottom surface. The top thermocouple measured the surface temperature.

The container was then placed in front of the open end of the channel. The fan and the heaters were turned on. The container was weighed at regular intervals to find the rate of drying and the average saturation. At the beginning and end of the drying runs, weighing was done more frequently than during the nearly constant drying rate period.

Due to the non-uniform flow distribution in the channel and the nonuniformity of heat generated by the heaters, the temperature distribution in the crosssection of the channel was not uniform (especially for low fan speeds). The temperature of the heated air was determined by taking the average of the surface temperature measured using dry beads.

The above experiment was done for the three predetermined fan settings. The experimental values of the drying rate, $\dot{m}_{\text {exp }}$, were compared with predicted values using two relationships - one based on mass нмт $30: 7-\mathrm{L}$ transfer and the other on heat transfer. Assuming that the heat and mass transfers are significant only at the surface, i.e. neglecting the porous medium surface temperature gradicnt and the internal vapor generation, the surface saturation coefficient can be determined from : (a) the analogy between mass and heat transfers (Section 2.1.5), (b) $\dot{m}^{*}=h_{\mathrm{h}}\left(T_{\mathrm{a}}^{*}-T_{\mathrm{l}}^{*}\right) / i_{\mathrm{fg}}^{*}$, and (c) the measured value of $\dot{m}_{\text {exp. }}^{*}$ The resulting equations are

$$
\begin{aligned}
\Psi=\frac{h_{\mathrm{h}}\left(T_{\mathrm{a}}^{*}-T_{1}^{*}\right) / i_{\mathrm{fg}}^{*}}{\frac{h_{\mathrm{h}}}{\rho_{\mathrm{sat}} \bar{M} c_{1}^{*}} M_{\mathrm{H}_{2} \mathrm{O}}\left(\rho_{\gamma, 1}-\rho_{\gamma, \mathrm{a}}\right) L e^{2 / 3}} \\
=\frac{\left(T_{\mathrm{a}}^{*}-T_{1}^{*}\right) \rho_{\mathrm{sat}} c_{1}^{*} \bar{M}}{\left(\rho_{\rho, 1}-\rho_{\rho, \mathrm{a}}\right) M_{\mathrm{H}_{2} \mathrm{O}} L e^{2 / 3} i_{\mathrm{fg}}^{*}}
\end{aligned}
$$

or

$$
\Psi=\frac{\dot{m}_{\exp }^{*}}{\frac{h_{\mathrm{h}}}{\rho_{\mathrm{sat}} \bar{M} c_{1}^{*}} M_{\mathrm{H}_{2} \mathrm{O}}\left(\rho_{\gamma, 1}-\rho_{\gamma, \mathrm{a}}\right) L e^{2 / 3}} .
$$

Note that equation (24a) is independent of $h_{\mathrm{h}}$ while equation (24b) is independent of $T_{\mathrm{a}}$. The magnitude of $\rho_{\gamma, \mathrm{a}}$ was determined using the surface temperature as the wet bulb temperature in order to find the relative humidity. Figure 4 shows the values of $\Psi$ determined using equations (24a) and (24b). The results show that relatively good agreement exists between the two predictions. This indicates that the measured heat transfer coefficients, the measured temperatures and the expression relating heat and mass transfer coefficients can be used with confidence.

The surface saturation coefficient is an indicator of the intensity of the mass transfer rate at the surface. After a short time, the surface is only partially covered by the liquid and also the surface is no longer planar. The rate of mass transfer depends on the surface geometry, surface saturation, and solid geometry, as well as the free stream velocity. Figure 5 shows how $\Psi$ changes with velocity (or $h_{\mathrm{h}}$ ) for the packed bed used. For the air heating arrangement used, the average temperature of the air passing over the packed bed did not remain constant as the velocity increased. This resulted in: $h_{\mathrm{h}}=30.9 \quad\left(T_{\mathrm{a}}=56\right), \quad h_{\mathrm{h}}=40.0$ $\left(T_{\mathrm{a}}=43.25\right)$ and $h_{\mathrm{h}}=51.9 \quad \mathrm{~W} \quad \mathrm{~m}^{-2} \quad \mathrm{~K}^{-1}$ $\left(T_{\mathrm{a}}=35.50^{\circ} \mathrm{C}\right)$. The results of ref. [6] for a certain wood (hygroscopic) are also shown. These results are based on a normalized average saturation, and are given here for reference only. Presently, no analysis of the relationship $\Psi=\Psi\left(s_{1}, R e, L e\right.$, geometry) is available.

\section{RESULTS AND DISCUSSION}

Since the analysis is based on the assumption of continuous liquid and vapor phases, the predicted results are for elapsed times up to the critical time. Beyond the critical time a method similar to that described in refs. $[3,11]$, which considers a two-phase 


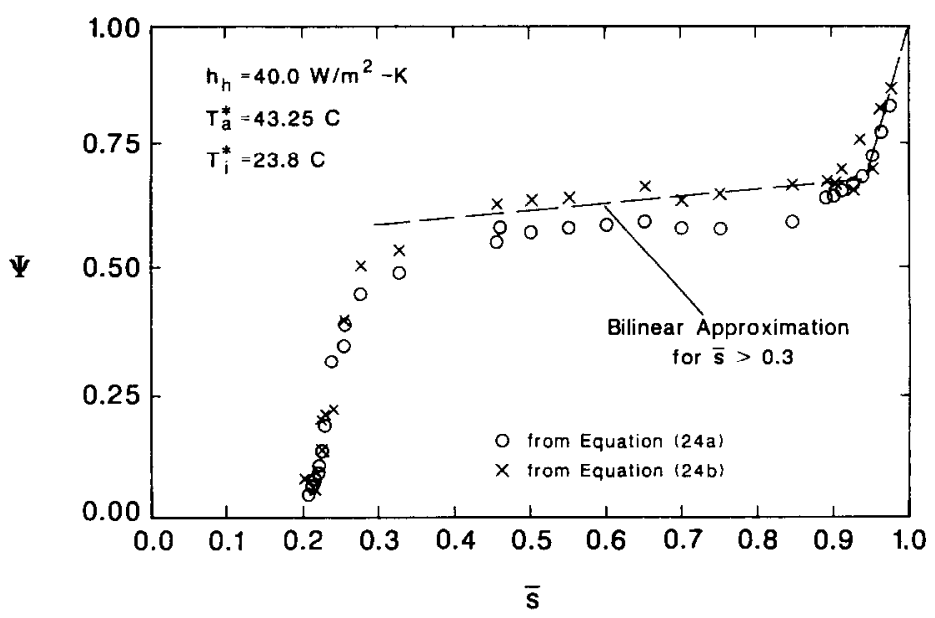

FIG. 4. The surface saturation coefficient as a function of average saturation for a specific fan setting.

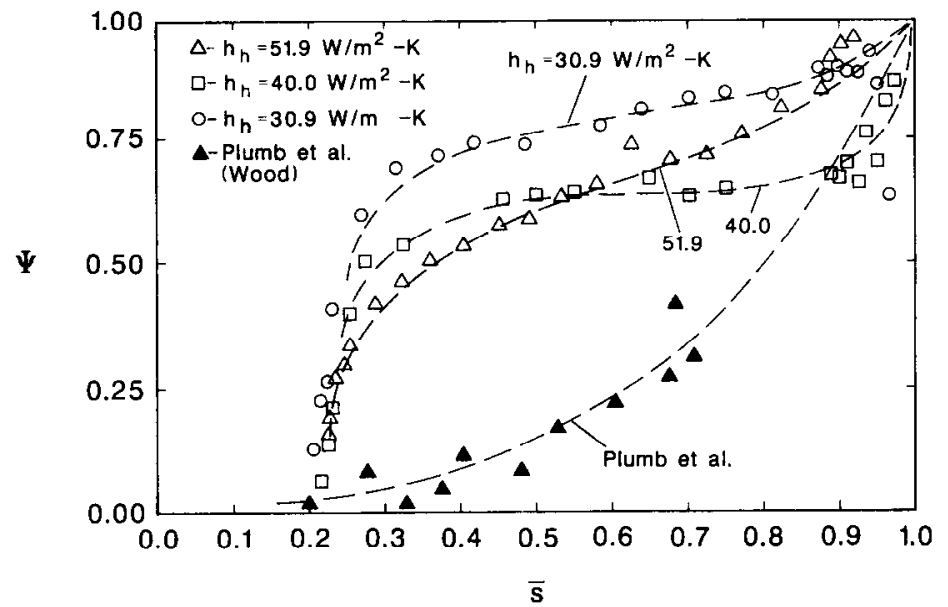

FIG. 5. The effect of velocity (or $h_{h}$ ) on the surface saturation coefficient. The results for a specific wood [6] are also given.

zone separating the vapor and liquid zones, must be adapted. Figure 6 gives the measured and predicted variations of the surface mass transfer ratc (cvaporation rate), the surface temperature, and average liquid saturation, with respect to time, for specific external fluid dynamic and temperature conditions. The physical properties are given in Tables 3(a) and (b). The surface mass transfer rate is zero at $t=0$ and initially increases rapidly and then decreases and reaches a plateau before decreasing again when the critical time is passed. Due to the excessive computational time required, the predicted results are for $K=10^{-11} \mathrm{~m}^{2}$ while the measured results are for $K$ of approximately $5 \times 10^{-11} \mathrm{~m}^{2}$. The surface mass transfer rate drops rapidly after the critical time is reached while at the same time the surface temperature increases rapidly. The experimental results show that the critical time is approximately $900 \mathrm{~min}$, while the predicted value is about $810 \mathrm{~min}$. The agreement can be considered reasonable because, as will be shown, $t_{\mathrm{c}}^{*}$ decreases with a decrease in $K$ and the prediction uses smaller $K$ than that measured experimentally.

Figure 7 shows the predicted distributions of the liquid saturation and the temperature in the slab. The results are for $t^{*}=798 \mathrm{~min}$ and for the same conditions as those given in Fig. 6 . At this particular time the heat is being stored in the porous medium. This heat storage will be further intensified in the post critical time era and very high surface temperatures can be reached. If possible, it may be desirable to change the heat supplying mode to internal heat generation (e.g. $G$ due to microwave heating) in order to avoid the drop in efficiency of drying or possible damage to the rigid matrix.

Based on the relatively good agrecment found between the predicted and measured critical time, it becomes desirable to determine the relationship between $t_{\mathrm{c}}$ and the various parameters. Examination of the parameters appearing in the dimensionless governing equations, i.e. equations (6), (7), (9) and (10), show that the dominant internal parameter is the Péclet number and the dominant external par- 


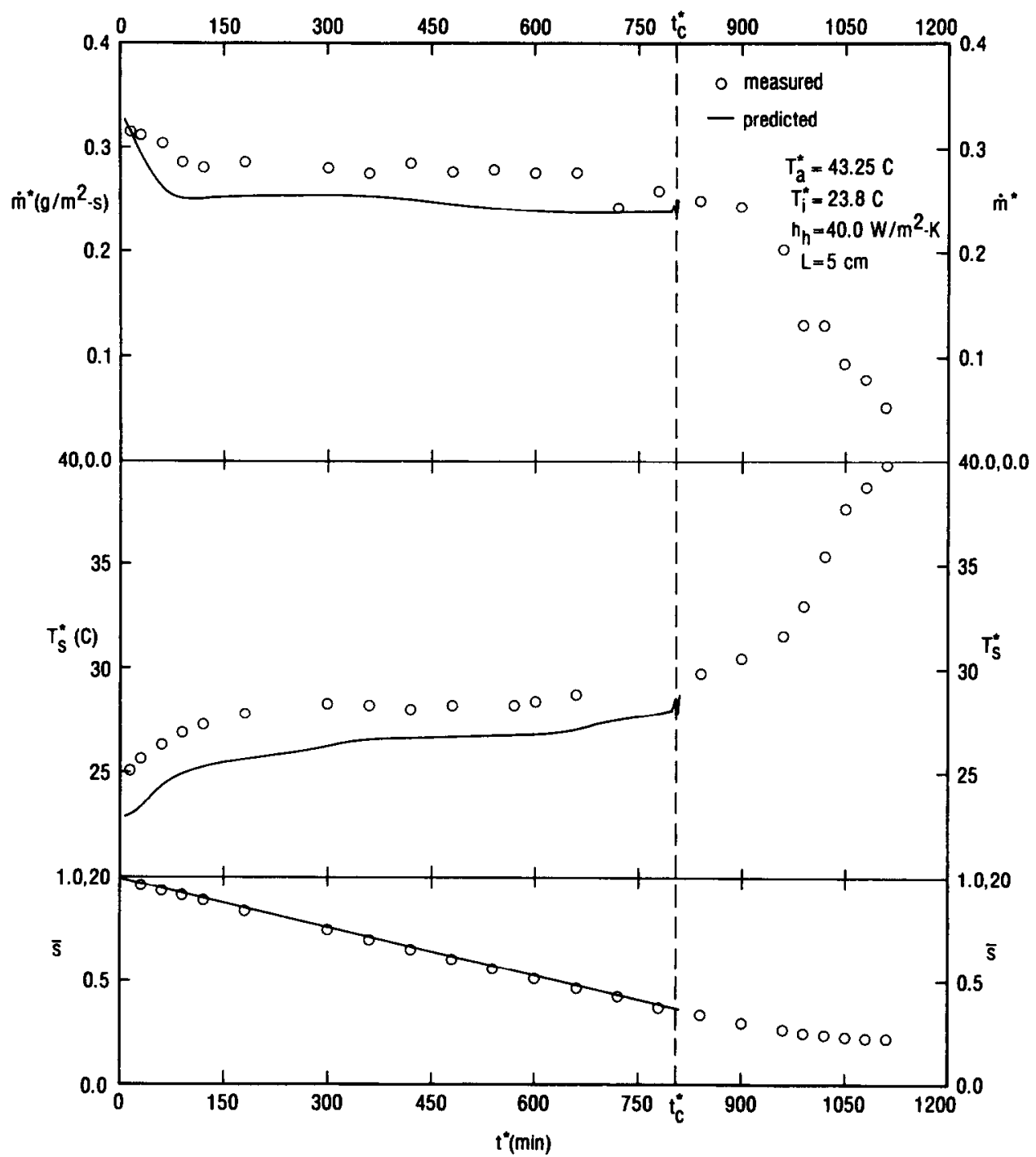

FIG. 6. Variations in drying rate, surface temperature and average saturation with respect to time. The circles show the experimental results and the solid lines show the predictions. The predictions are for up to the time of first appearance of dry patches on the surface (critical time).

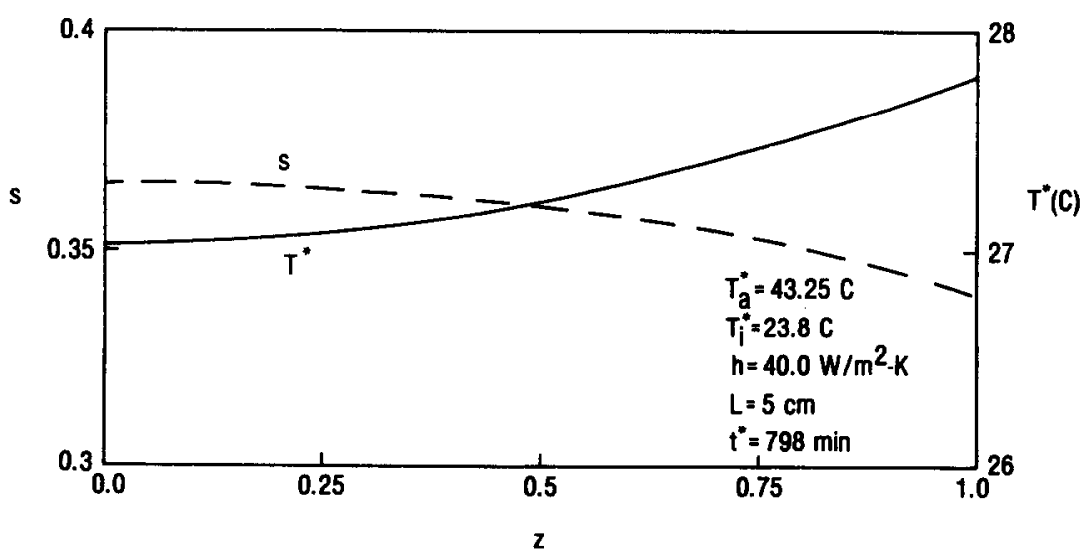

FIG. 7. The predicted saturation and temperature distribution in the slab for a specific setting. 

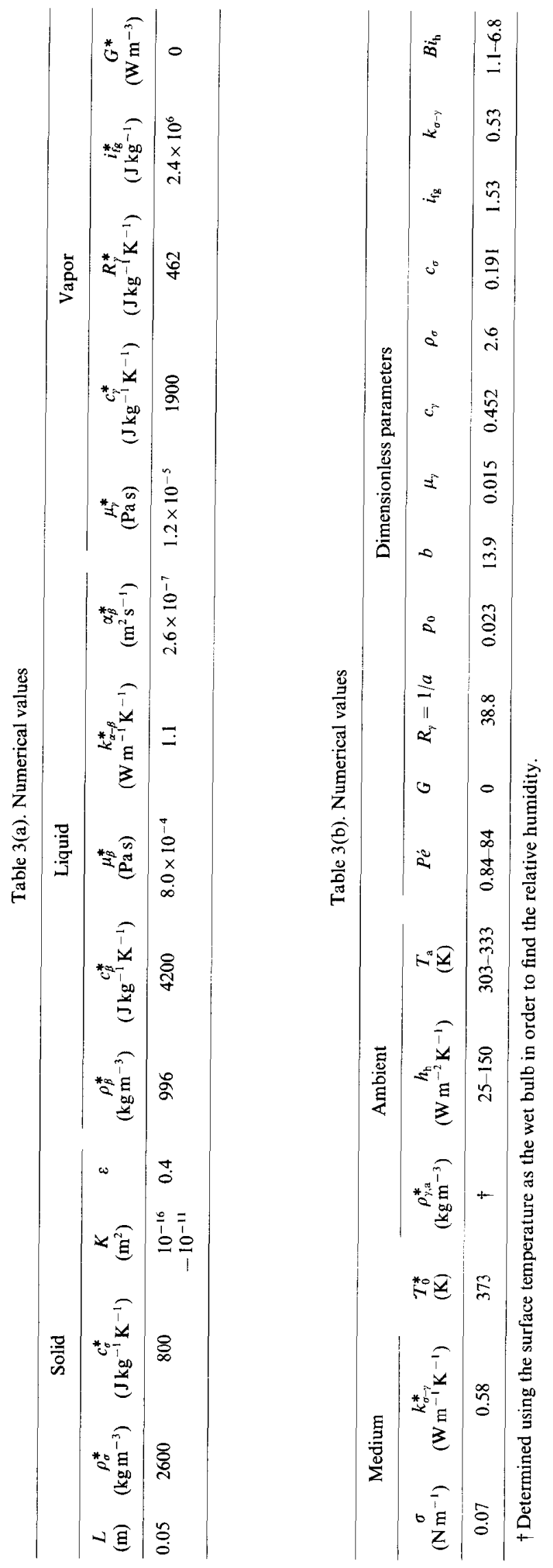


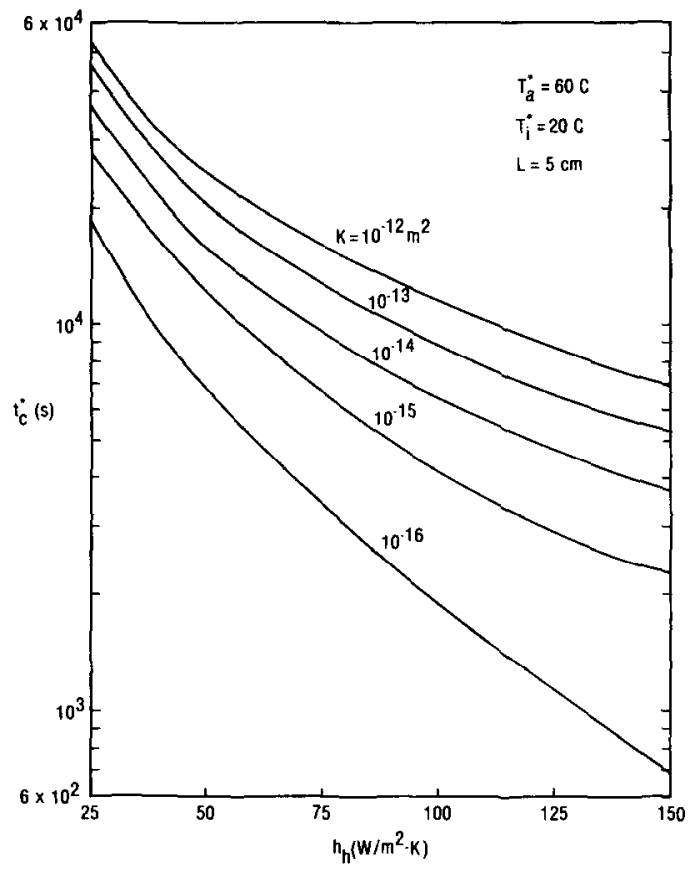

Fig. 8. Variations of the critical time with permeability and velocity (heat transfer coefficient) for a given ambient temperature and slab thickness.

ameters are $\Psi$ and $B i_{\mathrm{h}}$. Since these last two appear as a product in the surface mass transfer equation, then for media which are geometrically similar one may seek a relationship of the type $t_{\mathrm{c}}=t_{\mathrm{c}}\left(P \dot{e}, \Psi B i_{\mathrm{h}}\right)$.

Figure 8 shows the variation of $t_{\mathrm{c}}^{*}$ with $h_{\mathrm{h}}$ and $K$ for a given set of thermophysical properties, a layer length, a surface saturation coefficient $\Psi(s)$, and initial and ambient temperatures (Tables 3(a) and (b) and Fig. 4). As expected $t_{\mathrm{c}}^{*}$ decreases with increases in $h_{\mathrm{h}}$, i.e. the surface dries faster with an increase in the velocity. Also, as the permeability decreases, the liquid saturation gradient required for sufficient liquid flow to the surface increases. This results in a lower surface saturation which in turn reduces the relative liquid permeability and consequently the surface dries faster. Based on these behaviors, it is desirable to search for a relation of the type

$$
t_{c}=A \frac{P \dot{e}^{B}}{\left(\bar{\Psi} B i_{\mathrm{h}}\right)^{C}}
$$

where $A, B$, and $C$ are constant and

$$
\bar{\Psi}=\int_{0}^{1} \Psi \mathrm{d} \vec{s}
$$

(e.g. from Fig. 4).

However, due to the highly interactive heat and mass transfer processes, no single set of $(A, B, C)$ seems to apply over large ranges of $h_{\mathrm{h}}$ and $K$. For $K \geqslant 10^{-14} \mathrm{~m}^{2}$ and $h_{\mathrm{h}} \leqslant 100 \mathrm{~W} \mathrm{~m}^{-2} \mathrm{~K}^{-1}$, a set of coefficients was found to correlate the predicted results (for the specific case considered) to within $5 \%$.
Based on this correlation, we can extrapolate the predicted $t_{\mathrm{c}}^{*}$ for the conditions given in Fig. 6. Then the predicted $t_{c}^{*}=810 \mathrm{~min}$, which was for $K=10^{-11}$ $\mathrm{m}^{2}$ used in the computation, will become $t_{\mathrm{c}}^{*}=949 \mathrm{~min}$ for $K=5 \times 10^{-11} \mathrm{~m}^{2}$. Since the measured $t_{\mathrm{c}}^{*}$ is about $910 \mathrm{~min}$, this shows that the agreement between the measured and predicted $t_{\mathrm{c}}{ }^{*}$ is good. It is possible to find other $(A, B, C)$ that can be applied over other ranges of $h_{\mathrm{h}}$ and $K$. This was not attempted.

In ref. [6], it was found that for a specific solid matrix considered and using some specific empirical transport equations, and also for a given external conductance, the drying time (elapsed time up to the start of the pendular state) was nearly a constant for very low permeabilities (referred to as internally controlled) and also for very high permeabilities (externally controlled). Examination of the results given in Fig. 8 shows that no such trend is found for the cases considered and that one may categorize these cases in terms of belonging to a range of intermediate permeabilities, i.e. the transitional regime. However, as was discussed before, the critical time depends on both the intcrnal and extcrnal conductances.

\section{SUMMARY}

Convective drying of an initially liquid saturated slab is considered. The time at which the surface begins to dry is predicted using the available conservation equations and the experimentally determined constitutive relations. The predicted results are in good agreement with the experimental results. The critical time is proportional to the internal liquid transport conductance (the Péclet number) and inversely proportional to the external (surface) heat and mass transport conductance (the product of the surface saturation coefficient and the heat Biot number). The functional relationship, between the critical time, Péclet number, and Biot number, is not simple due to the rather complex interactions between the external and internal processes.

Acknowledgments-We would like to thank the reviewers for their constructive comments.

\section{REFERENCES}

1. A. V. Luikov, Heat and Mass Transfer in Capillaryparous Bodies. Pergamon Press, Oxford (1966).

2. H. F. Porter, P. Y. McCormick, R. L. Lucas and D. E. Wells, Gas-solid systems. In Chemical Engineers' Handbook (Edited by R. H. Perry and C. H. Chilton), 5th edn. McGraw-Hill, New York (1973).

3. S. Whitaker and W. T.-H. Chou, Drying granular porous media - theory and experiment, Drying Technol. 1, 3-33 (1983).

4. S. Whitaker, Heat and mass transfer in granular porous media. In Advances in Drying (Edited by A. S. Mujumdar), Vol. 1, pp. 23-61. Hemisphere, Washington, DC (1980). 
5. M. Fortes and M. R. Okos, Drying theories: the bases and limitations as applied to food and grains. In Advances in Drying (Edited by A. S. Mujumdar), pp. 119-154. Hemisphere, Washington, DC (1980).

6. O. A. Plumb, G. E. Spolek and B. A. Olmstead, Heat and mass transfer in wood during drying, Int. J. Heat Mass Transfer 28, 1669-1678 (1985).

7. D. Berger and D. C. T. Pei, Drying of hygroscopic capillary porous solids-a theoretical approach, Int. J. Heat Mass Transfer 16, 293-302 (1973).

8. T. Z. Harmathy, Simultaneous moisture and heat transfer in porous systems with particular reference to drying, I \& EC Fundamentals 8, 92-103 (1969).

9. C. L. D. Huang, Multi-phase moisture transfer in porous media subjected to temperature gradient, Int. J. Heat Mass Transfer 22, 1295-1407 (1979).

10. R. W. Lyczkowski and Y.-T. Chao, Comparison of Stefan model with two-phase model of coal drying, Int. $J$. Heat Mass Transfer 27, 1157-1169 (1984).

11. K. S. Udell, An exact solution to high temperature dry- ing of high permeability capillary porous media, ASME Paper No. 85-HT-66 (1985).

12. S. Whitaker, Simultaneous heat, mass and momentum transfer in porous media : a theory of drying, Adv. Heat Transfer 13, 119-203 (1977).

13. K. Udell and J. S. Fitch, Heat and mass transfer in capillary porous media considering evaporation, condensation and non-condensable gas effects. In Heat Transfer in Porous Media and Particulate Flows, ASME HTD-Vol. 46, pp. 103-110 (1985).

14. R. B. Bird, W. F. Stewart and F. N. I ightfont, Transport Phenomena, pp. 642-652. Wiley, New York (1960).

15. W. M. Kays and M. E. Crawford, Convective Heat and Mass Transfer, pp. 398-399. McGraw-Hill, New York (1980).

16. D. C. Reda and R. R. Eaton, Influence of steam/water relative permeability models on predicted geothermal reservoir performance: a sensitivity study, presented at the 6th Annual Workshop on Geothermal Reservoir Engineering, Stanford University (1980).

\title{
ETAT FUNICULAIRE DANS LE SECHAGE D'UNE COUCHE POREUSE
}

Résumé--Le séchage d'une couche poreuse non hygroscopique, initialement saturé par un liquide, est étudié expérimentalement depuis le temps de l'apparition des premières zones sèches (temps critique) en utilisant des lits de verre et le chauffage convectif, et analytiquement à partir des équations de conservation pour l'écoulement liquide poussé par capillarité et de relations empiriques. Un bon accord est trouvé entre les résultats calculés et l'expérience et les résultats montrent :

(a) Le transfert de masse surfacique dépend de la saturation superficielle. Ce coefficient de saturation superficielle qui est aussi fonction de la géométrie de surface et de la vitesse de l'écoulement libre, est déterminé expérimentalement.

(b) Le temps critique est un rapport de la conductance interne de transport liquide (nombre de Péclet) à la conductance externe de transport de vapeur (nombre de Biot).

\section{ZUR TROCKNUNG EINER PORÖSEN GESÄTTIGTEN PLATTE}

Zusammenfassung-Untersucht wird die Trocknung einer nichthygroskopischen porösen Platte, die zu Anfang mit Flüssigkcit gesättigt ist, bis zum Erscheinen von Austrocknungszonen (kritische Zeit), und zwar experimentell unter Verwendung von Glasperlen und konvektiver Beheizung und analytisch unter Verwendung der volumengemittelten Erhaltungsgleichungen für Kapillarströmung und empirischen Beziehungen. Es wird eine gute Übereinstimmung zwischen den berechneten und gemessenen Ergebnissen erzielt. Die Ergebnisse zeigen:

(a) Der Stofftransport an der Oberfläche hängt von der Oberflächensättigung ab. Dieser Oberflächensättigungs-Koeffizient, der auch eine Funktion der Oberflächengeometrie und der freien Strömungsgeschwindigkeit ist, wird experimentell bestimmt.

(b) Die kritische Zeit hängt vom Verhältnis des inneren Flüssigkeitstransport- (Peclet-Zahl) zum äußeren Dampftransport-Koeffizienten (Biot-Zahl) ab.

\section{КАНАТНОЕ СОСТОЯНИЕ ПРИ СУШКЕ ПОРИСТОГО СЛИТКА}

\begin{abstract}
Аннотацша-Экспериментально изучается сушка негитроскопичного пористого слитка, насыщенного жидкостью, до момента появления сухих участков (критическое время). Слиток состоял из стеклянных шарихов и конвективно нагревался. Теоретический анализ проведен путем осреднения по объему уравнений сохранения для капиллярного течения жидкости и использования эмпирических зависимостей. Найдено хорошее соответствие между результатами расчетов и измерений. Показано, что (а) интенсивность поверхностного массопереноса зависит от козффициента насыщения поверхности, который зависит от геометрии поверхности и скорости свободного потока и определяется экспериментально; (6) критическое время прсдставляет собой отношенне внутренней проводимости жидкости (число Пекле) х внешней проводимости пара (число Био).
\end{abstract}

